\title{
Das gedruckte Wort in Asien
}

\author{
von James Kottoor
}

\section{Asiens cbristlicher Journalist}

Jedes dieser drei Wörter bietet Stoff zum Nachdenken. Unser Hauptaugenmerk, Gegenstand unserer Ubberlegungen und unser Arbeitsgebiet ist Asien. Die erste Forderung ist deshalb, Asien zu begreifen.

Was ist Asien? Geschichtlich ist es ein Kontinent sehr alter Kulturen, die Heimat von Weltreligionen wie Buddhismus, Hinduismus, Konfuzianismus usw. Geografisch ist Asien der Welt größter Kontinent. Wirtschaftlich ist es ein Gebiet der Armut, Arbeitslosigkeit und Unterentwidklung. Politisch ein Mosaik aller möglichen Regierungsformen, die von der Diktatur bis zur Demokratie reichen. Demografisch ist es die Heimat zweier Drittel der Weltbevölkerung, die in einem beängstigenden Tempo explodiert. Kulturell ist es eine Sammlung von Nationen, die in ihrem Wachstum durch die westliche Kolonialisierung und Ausbeutung verkümmert sind.

Was ist ein Journalist? Er ist der Lieferant von Nachrichten und Meinungen in der Presse, die nur einen Teil im weiten Komplex der Massenkommunikation ausmacht. Sein Zielpublikum wird bestimmt und begrenzt durch die Art der Publikation, für die er arbeitet: Tages-, Wochen- oder Monatszeitung, internationales, nationales oder regionales Blatt, „weltliche“ oder kirchliche Publikation. Wenn es ein "weltliches“ Blatt ist, stellt sich die Frage, ob es politisch, wirtschaftlich, kulturell, wissenschaftlich oder pädagogisch ausgerichtet ist, bei einer religiösen Zeitung fragt sich, ob sie sich mit einer bestimmten Religion oder mit allen Religionen befaßt. Diese verschiedenen Faktoren bestimmen die Arbeit des Journalisten und setzen ihr Grenzen.

Man könnte mit Wilbur Schramm streiten, ob Massenkommunikation (in unserem Fall Journalismus) eine Profession sei wie die eines Rechtsanwalts, Arztes, Pfarrers, Lehrers oder Ingenieurs. Kein Zweifel besteht aber darüber, daß Massenkommunikation eine hohe Auffassung vom öffentlichen Dienst und darum auch eine hohe Berufsqualifikation für die Erfüllung dieser Aufgabe verlangt. Deshalb wird heute dem Verantwortungssinn bei den einzelnen Kommunikatoren großer Wert beigelegt, seien sie Schreiber, Schauspieler oder Rundfunk- und Fernsehleute. Dem klar hervortretenden Berufsethos muß sich jeder Kommunikator stellen, auch jeder Journalist in Asien. $\mathrm{Ob}$ und wieweit die Forderungen dieses Konzepts von den Journalisten Asiens erfüllt werden, muß von ihnen selbst beantwortet werden. Eine endgültige Antwort wird erst möglich sein, wenn Untersuchungen vorliegen, 1. über die Art und Weise, wie Pressemedien heute in Asien als Dienstleistung und Industrie existieren und funktionieren; 2. über die Anzahl der haupt- und nebenberuflich tätigen Journalisten in Asien; 3. über ihre nationalen oder internationalen Berufsvereinigungen und

Dr. James Kottoor, jetzt Bombay, war bis 1973 Chefredakteur der katholischen Wochenzeitung "New Leader" in Madras und bis 1975 im "Ecumenical Christian Centre", Whitefield (Bangalore), Indien, tätig. 
Organisationen; 4. über ihre allgemein akzeptierten Ziele, Ideale, Berufsqualifikationen und ethischen Richtlinien; und schließlich 5. über die heutigen Bedürfnisse der asiatischen Länder im Bereich der Presse in der Rangfolge der Wichtigkeit. Bisher ist meines Wissens eine solche Studie noch nicht vorgelegt worden.

Was ist ein christlicher Journalist? Hier muß zunächst gesagt werden, daß es so etwas wie christlichen Journalismus nicht gibt, ebensowenig wie christliche Arithmetik oder christliche Medizin und Technik. Diese Berufs- oder Wissenschaftszweige funktionieren unabhängig von der religiösen Einstellung dessen, der sich mit ihnen befaßt. So wird also hier gefragt nach einem Journalisten, der Christ, oder einem Christen, der Journalist ist. Im ersten Fall ist "Christ“ ein Anhängsel zu Journalist, im zweiten wird "Journalist ${ }^{*}$ dem Christen hinzugefügt. Der erste Fall bedeutet eine Beleidigung für die Christlichkeit des Betreffenden, die zu einem Zusatz, so wie etwa Sonntagschristentum, gemacht wird; der andere Fall ist eine Beleidigung des Journalistenberufs. Was ist also ein christlicher Journalist? Ich möchte ihn beschreiben als jemand, der zugleich und in vollem Sinn sowohl Christ als auch Journalist ist, oder als jemand, der im Journalistenberuf das beste Mittel sieht, seinen christlichen Auftrag auszuführen. Das setzt ein zweifaches Wissen voraus und eine zweifache Treue: Treue gegenüber den christlichen und Treue gegenüber den journalistischen Prinzipien.

Wenden wir uns nun nach dieser Klärung dem engeren Kreis der Probleme und Fragen christlicher Journalisten in Asien zu. Ich sagte ,engeren Kreis', weil die Christen in Asien - außer auf den Philippinen - eine Minderheit bilden. Zunächst ist auszumachen, wo die christlichen Journalisten und Schreiber $\mathrm{zu}$ finden sind: Arbeiten sie bei weltlichen Presseorganen oder Verlagshäusern, oder bei religiösen Zeitungen und Verlagen, oder sind sie unabhängige Verleger von Zeitungen und Büchern, oder sind sie einfach freiberuflich tätig? Sodann: Wie hoch ist ihre Zahl in jedem der asiatischen Länder? Schließlich: Gibt es irgendeine Form der Zusammenarbeit $\mathrm{zwischen} \mathrm{ihnen;} \mathrm{was} \mathrm{könnte} \mathrm{getan} \mathrm{werden,} \mathrm{um} \mathrm{eine} \mathrm{solche} \mathrm{Zusammenarbeit}$ zu stärken, wo sie besteht, oder wie könnte man sie beginnen, wo sie noch nicht vorhanden ist? Antworten auf diese drei Fragen zu finden ist eine riesige Aufgabe. Doch ich glaube, daß diese grundlegenden Fakten erst einmal zusammengetragen werden müssen, ehe irgendetwas Nützliches für die christlichen Kommunikatoren in den gedruckten Medien Asiens getan werden kann.

In Indien hat die katholische Kirche eine Untersuchung der katholischen Presse durchgeführt und die Ergebnisse 1972 in Buchform veröffentlicht ${ }^{1}$. Für den Anfang könnten auch für andere Kirchen regional entsprechende Studien durchgeführt werden, solange eine gemeinsame Untersuchung auf nationaler Ebene noch nicht möglich ist. Wie könnte das im einzelnen aussehen? Vielleicht können die Hauptpunkte der Untersuchung über die katholische Presse Indiens als Anhaltspunkte dienen. Diese Untersuchung umfaßte 143 katholische Periodika, 108 Herausgeber, 263 Schreiber und Journalisten und 70 Pressehäuser. Untersucht wurden 2 Tages-, 12 Wochenzeitungen, 2 vierzehntäglich erscheinende Publikationen, 95 Monats-, 13 Vierteljahresschriften und andere.

\section{Das Publikum des christlichen Journalismus}

Das Zielpublikum für das Apostolat der Presse in Asien ist die gesamte Bevölkerung dieses Kontinents, 2.250 Millionen Menschen, die fast zwei Drittel der Weltbevölke- 
rung ausmachen. Von diesen sind 46,8\% Analphabeten, 40\% unter 15 Jahre alt. Das Pro-Kopf-Einkommen beträgt 340 Dollars (das von Neuseeland und Australien eingeschlossen) - das der USA liegt vergleichsweise bei 4.760. Pro Person stehen knapp 3.000 Quadratmeter Adkerland zur Verfügung (um zu überleben, braucht ein Mensch gut 10.000 Quadratmeter Land pro Jahr).

Nach dem Manorama Year Book 1974 gab es 1973 in Asien 469.990.000 Hindus, 331.115.000 Muslims, 304.300.000 Konfuzianer, 301.058.000 Buddhisten und 76.468.000 Christen (davon entfallen auf die Philippinen allein über 32 Millionen), 60.000.000 Shintoisten, 51.250.000 Taoisten, 2.760.600 Juden und andere religiöse Gruppen.

Vom journalistischen Standpunkt aus kann diese asiatische Bevölkerung in zwei Hauptgruppen unterteilt werden: 1. Christen, 2. Nichtchristen. Die zweite Gruppe kann wiederum dreifach unterteilt werden: in 1. Glaubende (die Anhänger der verschiedenen Religionen in Asien), 2. Menschen guten Willens oder Wahrheitssucher (die keiner Religion besonders verbunden sind, sich aber von humanitären Prinzipien leiten lassen), 3. Atheisten oder Nichtglaubende (wegen theoretischer oder praktischer Gründe).

Angesichts dieser vielfältigen Gruppierungen ist $\mathrm{zu}$ fragen:

1. Wer bildet bisher das Publikum der christlichen Presse?

2. Wer sollte Adressat der christlichen Presse sein?

Auf die erste Frage läßt sich antworten, daß bisher nicht die gesamte christliche Gemeinschaft dieses Publikum bildet, sondern kleine Gruppen von konfessionell getrennten Christen. Dies macht schon ein grober Uberblick deutlich, wenn man den Inhalt der Publikationen betrachtet, die von einem ausgeprägten "nationalen und konfessionellen Kirchturmdenken ${ }^{\alpha}$ geprägt sind, wie Lee Soo Jin, Sekretär der Abteilung Kommunikation von CCA (Christian Conference of Asia) den Sachverhalt beschrieben hat. Wir haben immer noch keine Publikation, die über diese konfessionell geprägte Gettomentalität hinausreicht. Dies ist die unglückselige Konsequenz des Argernisses einer getrennten Christenheit, deren Erben wir alle sind. Aber wir sollten die Situation nicht so belassen, wie wir sie vorgefunden haben. Die Antwort auf die zweite Frage klärt sich somit auch. Das Publikum der christlichen Presse sollte nicht durch diese engen Kirchturm-Grenzen bestimmt werden, nicht einmal durch die weitere Grenze aller Christen, sondern es müßte alle Menschen umfassen, Glaubende wie Atheisten. Warum? Weil Christen, die sich ausschließlich mit Christen befassen, gegen Jesus Zeugnis ablegen, dessen Ziel alle Menschen und der ganze Mensch waren. Nur ein Weg, der alle Teilungen überwindet, kann in voller Weise dem entsprechen, was OKumenismus meint. Sind unsere Kirchen, vor allem die Journalisten in Asien, für diese Herausforderung bereit?

\section{Evangelisation durch Presse und Schrifttum}

Evangelisation, die darauf zielt, Menschen zu helfen, Christus als den Herrn zu bekennen, setzt Verkündigung und deshalb auch Verkündiger voraus (vgl. Röm 10,13-15). Aber was hat Journalismus mit Verkündigung zu tun? Ist die Presse ein Mittel, Christus zu verkaufen?

Hier sollte zwischen Verkündigung und Kommunikation unterschieden werden. Das Wort Verkündigung (preaching) ist, wegen des darin enthaltenen Propagandatons 
und der damit verbundenen Verkaufsassoziationen, in Mißkredit geraten. Niemand möchte bepredigt werden, und das aus gutem Grund. Uber den Begriff ,Kommunikation' ist niemand ungehalten, wenngleich die Kommunikationsmedien hinterlistiger gehandhabt werden können als Verkündigung im schlechten Sinn, z. B. durch Manipulation. Wer sind denn die wirklichen Prediger heute? Ich denke hier nicht an die Predigten in Kirchen, Kapellen oder christlichen Versammlungen, sondern an die Predigt, die in aller Offentlichkeit stattfindet, in einer Welt, die durch die Massenmedien zu einem globalen Dorf reduziert worden ist. Prediger sind hier diejenigen, die die Massenmedien - vor allem Presse, Radio, Film und Fernsehen - leiten, kontrollieren oder sogar manipulieren. Was ist der christliche Anteil an diesem weiten Kommunikationsnetz, vor allem in Asien? Sehr oft werden diese Sender von Leuten besetzt und geleitet, die Nicht-Christen, unchristlich oder gar anti-christlich eingestellt sind. Und doch hört die ganze Welt, die Christen eingeschlossen, diesen Medien aufmerksam zu.

Das ungeheure Wachstum der Massenmedien hat sogar aus Christen, die nach göttlichem Auftrag doch Verkünder des Wortes sein sollten, Hörer der Verkündiger der weltlichen Welt gemacht. Die Christen von heute haben ihre traditionelle Rolle verloren, im richtigen Sinn zu predigen, das heißt mit der Welt, und weltlichen Schaltstellen, zu kommunizieren. Das gilt auch für Asien. Mit anderen Worten, in diesem Zeitalter der Massenmedien kann die gute Nachricht nicht gepredigt oder vermittelt werden, d. h. Evangelisation ist unmöglich ohne die Hilfe der Kommunikationsmedien. Deshalb haben wir ein Recht, von der Evangelisation durch die Presse zu sprechen.

Welche Art von Evangelisation können wir durch das gedruckte Wort leisten? Hier sollte zunächst der Begriff ,Evangelisation' geklärt werden. Im strengen Wortverstand meint er, die Frohe Botschaft jenen zu bringen, die sie nie vorher vernommen haben, oder denen, die sie zwar gehört haben, aber noch nicht als Christen getauft wurden. Dann wird dieser Begriff auch gebraucht für die Unterweisung der schon Getauften, um ihren Glauben zu vertiefen und zu stärken. Letzteres ist die Aufgabe, die schon ,Bekehrten' zu bekehren oder den Evangelisator zu evangelisieren, was genau Katechese zu nennen wäre. Beides ist notwendig, Evangelisierung im strengen Sinn und die Evangelisierung der schon Evangelisierten (Katechese). Beides kann und muß durch die Presse geschehen. Unsere Aufgabe ist es, den Vorrang festzulegen.

Evangelisierung des Evangelisators: Nehmen wir unsere christlichen Presseorgane in Asien, so können wir mit einiger Sicherheit annehmen, daß sie sich im allgemeinen an Christen richten und sich darum bemühen, die Evangelisatoren $\mathrm{zu}$ evangelisieren. Ist das aber die dringlichste Aufgabe der Kirchen im heutigen Asien? „Die Kirche ist ein Zeichen des Evangeliums; sie kann aber auch ein Gegen-Zeichen und ein GegenZeugnis sein, das das rechte Verständnis der evangelischen Botschaft blockiert, und manchmal ist sie das auch. Viele Menschen und Gruppen, vor allem junge Arbeiter, sehen die institutionelle Kirche als Hindernis für ihren Glauben an Jesus Christus und sein Evangelium. Da versagt die Kirche, ein glaubwürdiges Zeichen zu sein für das, was sie verkündet; sie entfremdet viele vom Glauben. Die Kirche muß daher - als Institution - zum Evangelium zurückkehren, muß selbst evangelisiert werden." (Fr. Tissa Balasuriya, bei der Internationalen Theologenkonferenz von Nagpur, 1972). Wir können sagen, daß diese Notwendigkeit auch heute noch besteht: Christen zu evangelisieren, die nur dem Namen nach Christen sind; unsere Pfarrer und Priester, die nicht von der Verkündigung, sondern von der Karriere bestimmt wer- 
den; unsere Bischöfe, die vielleicht mehr mit Macht, Stellung und Ansehen befaßt sind als mit Christus und seinem Reich; evangelisiert werden müssen auch Organisationen wie nationale Kirchenräte oder Bischofskonferenzen, der CCA, der WCC, die Weltsynode der Bischöfe, der Vatikan usw.

Den Evangelisator zu evangelisieren bedeutet - noch genauer -, daß die Presse die Funktionen eines Stärkungsmittels (tonic) und einer Schutzimpfung (vaccination) übernehmen muß. ,Stärkungsmittel' bedeutet, daß kräftigende Gedankennahrung zur Verfügung gestellt werden muß, wie Informationen über theologisches Denken zu allen Fragen, die das Leben der Christen in Asien betreffen und ihnen helfen können, Gründe zu finden für das, was sie als Christen tun; die ihnen helfen, aus Überzeugung zu handeln und nicht aus Zwang, Routine, Gewohnheit oder Tradition, kurzum die die Kirche in Asien aufbauen, nicht mit Steinen und Mörtel, sondern im Geist und Herzen der Christen.

Die Funktionen einer Schutzimpfung zu übernehmen bedeutet, die Christen in Asien gegen Schocks zu immunisieren, das heißt: sie schockfest zu machen gegen umstürzlerische und aufrührerische Fragen über Gerechtigkeit, Befreiung und Entwicklung in der Kirche und in Asien; fest im Dialog mit Atheisten und Kommunisten in der Erfahrung eines weltlichen oder religionslosen Christentums, eines nach-ökumenischen Christentums, der Gott-ist-tot-Theologie usw. Grundlegende Fragen aus diesen Bereichen sollten in den Medien offen diskutiert werden, in einer Atmosphäre der Freiheit und nicht der Angst, um die Menschen zu unterweisen, um sie am Heilsgeschehen in Asien aktiv teilnehmen zu lassen; um unsere Christen fähig zu machen, sich ihre eigenen Wertvorstellungen, Urteile und Überzeugungen zu bilden, die auf Vernunft beruhen und nicht auf Leidenschaft, so daß sie bei ideologischen Auseinandersetzungen auf eigenem, festem Grund stehen können, bei jenem Kampf um die Herzen der Menschen, der in Asien im Gange ist; damit Christen und ihre Führer nicht in Elfenbeintürmen leben mit dem Bewußtsein, daß alles in Ordnung sei, sondern damit sie mit den harten Realitäten der zweifelnden Tomasse, der verleugnenden Petrusse, der fragenden Intellektuellen, der Jugend in der Kirche in Berührung kommen, und mit denjenigen, die die Kirche verlassen haben und nicht mehr weiter mit ihr gehen wollen. So könnten die Christen demütig und einfach leben, nicht vertrauend auf irdische Sicherheiten, die sie selbst gebaut haben, sondern im Vertrauen auf Christus, der seinen Sieg gründete auf die Annahme der schmerzhaften Unsicherheit von Tod und Niederlage des Kreuzes, und - schlimmer noch der Unsicherheit, von allen - sogar von Gott - verlassen zu sein.

Die Evangelisation der christlichen Gemeinschaft auf den verschiedenen Ebenen Fragen, Herausforderung, Stärkung, Immunisierung - durch die Presse ist notwendig. Zu fragen bleibt jedoch, ob diese Art Evangelisation auch für Asien erforderlich ist, und wenn ja, ob wir diese Aufgabe in der Presse gut erfüllen können.

Die eigentliche Evangelisierung: Aber wie steht es mit der eigentlichen Evangelisierung, d. h., dem Erreichen derjenigen, die noch nicht an Christus glauben und in Asien die große Mehrheit ausmachen? Kann eine religiöse Presse, vor allem wenn sie offen christlich ist, die Aufmerksamkeit von vielen Millionen Asiaten, die tief in ihren alten Religionen verwurzelt sind, gewinnen und ihr Interesse wachhalten? Ich glaube nicht, daß eine religiöse Presse, viel weniger noch eine christliche Presse, geeignet ist, diese Millionen mit der Frohen Botschaft zu erreichen. Ich möchte sogar behaupten, daß die christliche Presse, wie sie heute ist, sterben muß, wenn sie ihre 
Aufgabe erfüllen will: dem ganzen Menschen und allen Menschen zu dienen. Ihre selbstgeschaffenen Grenzen, die religiöse, kirchliche, seelenrettende Mission muß sterben, damit sie am dritten Tag wieder auferstehen kann, um die weiterreichende Rolle und Aufgabe des Dienstes auch an den weltlichen, nationalen, regionalen und menschlichen Bedürfnissen der Entwicklungsländer in Asien zu übernehmen. Der Tod der christlichen Presse, von dem ich spreche, ist ein erlösender Tod, der umwandelt und zu einem erfüllteren und besseren Lebensstil führt, der für alle annehmbar ist, unabhängig von Kaste, Glaubensbekenntnis und Nationalität. Nur eine weltliche Presse kann diese Aufgabe erfüllen. Deshalb muß die christliche Presse weltlich werden, wenn sie ernsthaft das Werk der Evangelisierung im eigentlichen Sinn in Asien durchführen will. Die Gründe für meine Behauptung sind folgende:

\section{Warum weltlich werden?}

1. Aufgabe der christlichen Presse ist es, das lebenspendende Wort allen Menschen dieser Welt mitzuteilen. In Christus hat sich Gott selbst der Menschheit mitgeteilt. Christus ist Gottes Wort und Gottes Leben, oder - besser gesagt - das Wort, das Leben gibt. Aufgabe der christlichen Presse ist es, dieses Wort an jedem Ort ständlich zu machen. Wie kann sie aber dieses Wort ansprechend machen für alle Menschen, nicht nur für die Christen, wenn sie nicht weltliche Kleidung anlegt?

2. Entwicklung (Entwicklung ist der neue Name für Frieden, und Christus ist unser Friede), die die christliche Presse fördert und unterstützt, sollte auf das Wohl des ganzen Menschen und aller Menschen zielen. Dazu muß die christliche Presse dem religiösen, politischen, wirtschaftlichen, kulturellen, weltlichen und materialistischen Menschen neues Leben einhauchen - das wahre Leben, das vom Wort kommt -, weil Christus kam, um den Menschen mit sich zu versöhnen (und nicht, um ihn in sich bekämpfende Gruppen zu teilen), zu versöhnen auch mit Gott und seinem Nächsten. Diese Aufgabe zu erfüllen, erschien Christus unter uns wie einer von uns, um uns zu dem zu machen, was Er nicht zu sein schien - göttlich.

Ebenso muß die christliche Presse, wenn sie eben die gleiche Aufgabe Christi weiterführen will, wie er ihre überirdischen Gewänder ablegen und in die Welt treten als eine von vielen weltlichen Publikationen und allen Menschen alles werden, um alle zu retten.

3. Aufgabe der christlichen Presse sollte es sein, möglichst viele Menschen anzusprechen. Alles Menschliche und Rationale spricht an, wenn es in einem nicht-religiösen Kontext dargeboten wird. Wie kann aber die christliche Presse solches tun, solange sie in religiösen Gewändern einherkommt? Man sollte keine Angst darum haben, daß durch das Ablegen des christlichen oder religiösen Gewandes die christliche Presse ihre Identität verlieren oder ihr Ziel verfehlen würde. Das wird nicht der Fall sein, solange sie bestimmt sein wird von dem, was wirklich menschlich und vernünftig ist, weil das wirklich Menschliche und Vernünftige auch das wabrbaft Christliche ist.

4. Okumenische Überlegungen, die heute an Einfluß gewinnen, fordern uns auf, die Gettos unserer Kirchen, unseres konfessionellen Denkens und Handelns zu verlassen. Die Kirche muß dort sein, wo die Menschen sind - auf dem Marktplatz, vor allem der Entwicklungsländer, die die Wehen einer politischen, wirtschaftlichen und kulturellen Wiedergeburt durchleiden. Wie können die Kirchen in diesen Bereichen der Entwidklungsländer einen Ansatzpunkt, vielleicht sogar eine führende Stellung ge- 
winnen, wenn nicht durch eine mächtige weltliche Presse, um die verschiedenen Entwidklungsprobleme von einem menschlichen und vernünftigen Standpunkt aus zu behandeln?

5. Die öffentliche Meinung ist ein Faktor, mit dem wir uns sowohl in der Kirche wie im Staat auseinanderzusetzen haben. Die meisten Länder Asiens haben eine demokratische Verfassung, und Offentlichkeit und öffentliche Meinung spielen im Leben dieser Nationen auch weiterhin eine wichtige Rolle. Natürlich mag Pressefreiheit in vielen dieser Länder nur eine Farce sein. Wie aber kann die Kirche in den Ländern mit einer freien Presse ihre heilsame Rolle erfüllen, zugunsten der wahren Interessen dieser Länder die öffentliche Meinung zu bilden, wenn sie kein machtvolles Organ der öffentlichen Meinung besitzt, d. h., vor allem eine wirksame weltliche Presse, die allein die Führung dieser Länder nachhaltig beeinflussen kann?

6. Aufgabe des Christen ist es, wie Sauerteig zu wirken, d. h., von einer verborgenen und Minderheiten-Position aus. In vielen Entwicklungsländern Asiens sind die Christen eine Minderheit, und wir haben uns angewöhnt, den Staat andauernd zu bitten, uns zu schützen, zu unterstützen und zu bemuttern, weil wir uns von den Mehrheiten ungerecht behandelt fühlen.

Berufung des Christen ist es nicht, Mehrheit zu sein, sondern die kleine Herde, eine Minderheit wie die Hefe. Die Hefe sollte sich nicht beklagen, daß sie nicht Teig ist. Ihre Aufgabe ist es, ein kleiner Teil zu sein, der dennoch imstande ist, die große Masse zu verwandeln. Ebenso ist es die Aufgabe der Kirchen in Asien, machtvoller Verwandlungsfaktor zu sein in der Gesellschaft, in der sie leben. Wie aber kann die Hefe mit der Masse zusammengebracht werden, wenn nicht durch eine weltliche Presse, die ihr allein die Möglichkeit gibt, sich in die Masse der Entwicklungsprobleme dieser Länder einzugraben.

7. Einige Entwicklungsländer in Asien, wie das moderne Indien, werden immer feindseliger gegenüber allem, was missionarischen oder evangelistischen Aktivitäten ähnelt. Die Anti-Konversionsgesetze, die in verschiedenen Staaten Indiens eingebracht wurden, sind Zeichen einer allgemeinen Feindseligkeit und Opposition gegenüber allem, was irgendwie mit direkter Predigt $\mathrm{zu}$ tun hat. Christen dürfen das göttliche Gebot der Verkündigung nicht vernachlässigen, sie müssen predigen, wenn nicht direkt, dann indirekt. Es geht dabei nicht um den Namen "Christ" oder "Katholik“, sondern darum, die Lösungen Christi für die menschlichen Probleme des Hungers, der Krankheit, der Armut, der Ungebildetheit, der Ausbeutung, des Krieges zu vermitteln. Dies alles ist das himalaya-mächtige Problem der Entwicklung. Um den Widerstand von Nicht-Christen gegen ausgesprochen christliche Lösungen zu verstehen, sollten wir unsere eigene Opposition gegenüber erklärtermaßen nichtchristlichen Lösungen für christliche Probleme untersuchen. Wir schalten meistens $a b$, wenn wir hören, daß eine Lösung nicht christlich ist. Was anders können wir von einem Nicht-Christen erwarten, wenn wir ihm eine christliche Lösung für sein Problem anbieten? Der Grund für diesen Sachverhalt liegt vor allem darin, daß mittlerweile Begriffe wie "christlich" oder "nicht christlich" in allen praktischen Angelegenheiten etwas Sektiererisches an sich haben. Diese Fallgrube müssen wir vermeiden, wenn wir in einer weltlichen Zeitung unsere Lösungen als menschlich und vernünftig anbieten.

8. Die Notwendigkeit für eine von der Kirche unterstützte weltliche Presse ist drängender in den Entwicklungsländern Asiens als in den entwickelten Ländern des Westens. $\mathrm{Da}$ die meisten Länder des Westens eine christliche Tradition, christliches Erbe und eine große christliche Bevölkerung haben, können christliche Nachrichten 
und Meinungen relativ leicht durch die weltliche Presse vermittelt werden, was nicht der Fall ist in den Ländern Asiens, die in der Mehrheit nicht christlich sind und sogar mißtrauisch gegenüber allem, was irgendwie christlich aussieht. Die Folge ist, daß selbst bedeutende christliche Nachrichten teilweise oder ganz von den Zeitungsspalten ausgeschlossen bleiben. Deshalb kann die Aufgabe, die Kirche einer nicht christlichen Welt in Asien präsent zu machen, nur durch eine von der Kirche unterstützte - nicht von ihr kontrollierte - Presse gelöst werden. Sind wir in Asien bereit, unserer Presse eine neue Richtung zu geben, genauer: uns in einer weltlichen Presse mit der großen Masse der Nicht-Christen zu befassen, einer weltlichen, aber christlich inspirierten Presse, die ein Schritt hin zur Evangelisation ist?

Christen, die - ohne Beachtung von Grenzen für ihren Nächsten leben und arbeiten (Horizontalismus), müssen belebt und mit Energie gespeist werden von jenem Vertikalismus, den Christus im Vaterunser lehrt. Wie kann dieses Gebet ein ehrliches Zeichen werden für das, was es darstellt - die Vaterschaft Gottes und das Brüdersein aller Menschen - wenn nicht die Christen sich angetrieben fühlen, mit allen Menschen eins $z u$ werden?

Ideal und Realität sind zwei auseinandergerückte Pole. Aber wir sollten anfangen und hart arbeiten, die Kluft zwischen ihnen zu verkleinern.

Anmerkung:

1. Tomy Luiz SVD: Indian Catholic Press. A Survey. Bombay 1971; vgl. CS 6:1973, S. $160-163$.

\section{S U M M A R Y}

The Christian journalist in Asia has first of all to understand Asia. At the same time, he needs a clear understanding of the profession of a journalist. Together with this goes the conviction of being a christian. He should try to know his readership and should understand that there is, up till now, ghetto mentality of the christian press that should be overcome. The author is of the opinion that the christian press in its present stage in Asia, has somehow to die and rise again to fulfill its purpose to serve the total man. He pleas for a secular press inspired by christianity as a step towards Evangelization.

\section{RESUME}

Le journaliste chrétien en Asie doit d'abord apprendre à comprendre l'Asie. Il doit se faire une image claire de son métier de journaliste. Et enfin, il doit pénétrer le sens du christianisme pour être un journaliste dhrétien en Asie. Il doit apprendre à connaître ses lecteurs, et il doit comprendre que la presse chrétienne doit surmonter la mentalité de guetto à laquelle elle était habituée jusqu'à présent. L'auteur est d'avis que la presse chrétienne en Asie, telle qu'elle est aujourd'hui, doive mourir et réssusciter comme neuve afin de remplir sa tâche qui est de servir tout l'homme et tous les hommes. Il plaide pour une presse laïque mais d'inspiration chrétienne qui serait un pas en direction de l'évangélisation.

\section{R E S U M E N}

El periodista cristiano en Asia debe aprender ante todo a conocer Asia. Debe hacerse una idea de la imagen profesional del periodista y debe enfín profundizar el sentido del cristianismo para ser en Asia un periodista cristiano. Debe conocer su público y ser consciente de que la prensa cristiana debe superar la mentalidad de getto cultivada hasta ahora. El autor opina que la prensa cristiana en Asia, tal como ahora existe, debe desaparecer y surgir de nuevo si quiere cumplir el cometido de servir a todo el hombre y a todos los hombres. Aboga por una prensa mundana pero inspirada en el cristianismo que sea un paso hacia la evangelización. 